\title{
EMISIÓN DE METANO ENTÉRICOEN RUMIANTES EN PASTOREO
}

\author{
ENTERIC METHANE EMISSIONBY GRAZING RUMINANTS
}

\author{
Vargas, J. ${ }^{1}$, E. Cárdenas ${ }^{1}$, M. Pabón ${ }^{1,2}$ y J. Carulla ${ }^{1 *}$
}

\begin{abstract}
${ }^{1}$ Grupo de Investigación en Nutrición Animal. Departamento de Producción Animal. Facultad de Medicina Veterinaria y de Zootecnia. Universidad Nacional de Colombia. Bogotá. Colombia.

${ }^{2}$ Departamento de Química. Facultad de Ciencias. Universidad Nacional de Colombia. Bogotá. Colombia. *jecarullaf@unal.edu.co
\end{abstract}

\section{Palabras clave adicionales}

Forrajes. Gases efecto invernadero. Sistemas pastoriles. Fibra. Tasa de paso. Metabolitos secundarios. Consumo voluntario.

\section{RESUMEN}

Esta revisión explora el conocimiento asociado a los aspectos que determinan la emisión de metano en rumiantes en pastoreo. Se aborda el efecto de la especie forrajera, variedades dentro de especies y asociaciones gramínea/leguminosa, además de aspectos de manejo de la pastura como su madurez, carga animal, sistema de pastoreo y nivel de fertilización. El análisis hace énfasis en las influencias en las emisiones por unidad de materia seca consumida. Existen diferencias entre especies forrajeras en la emisión de metano que están asociadas a diferencias en las concentraciones de fibra, a variaciones en la tasa de paso y la presencia de metabolitos secundarios. Mayores concentraciones de fibra reducen las tasas de paso, el consumo de materia seca y aumenta la producción de metano por unidad de materia seca consumida. El efecto de la inclusión de leguminosas sobre la producción de metano es variable y esta influenciado por la presencia de metabolitos secundarios como los taninos y las saponinas. Los resultados de los trabajos revisados sugieren que existen oportunidades para reducir las emisiones de metano por unidad de materia seca consumida cuando las prácticas de manejo de las pasturas aumenten las tasas de paso y el consumo voluntario. Además el aumento en el consumo conllevaría a la reducción de emisiones de metano por unidad de producto animal.

\author{
AdDitional KEYWORDS \\ Greenhouse gases. Grasslands. Grazing sys- \\ tems. Fiber. Rate of passage. Secondary \\ metabolites. Voluntary intake.
}

\section{SUMMARY}

This review explores dietary and feeding aspects that influence methane emissions by grazing ruminants. The effects of forage species, varieties within species and grass-legume pastures are discussed. Aspects related to pasture management such as maturity, stocking rate, grazing system and fertilization are also reviewed. Differences within forage species in methane emissions are associated to fiber concentration, rate of passage and presence of secondary metabolites. Higher fiber concentrations reduce passage rates, dry matter intake and increase methane production per unit of dry matter intake. The effect of legume inclusion on methane production is variable and it is influenced by the presence of second metabolites such as tannins and saponins. Opportunities do exist to reduce methane emission per unit of dry matter intake when pasture management practices increase rates of passage and voluntary intake, which also will translate in reduced emissions per unit of animal product.

\section{INTRODUCCIÓN}

La emisión de metano entérico representa un $30 \%$ de las emisiones de metano de origen antropogénico y un $50 \%$ de las provenientes del sector agrícola (Solomon et al., 2007). El metano es un gas de efecto 
invernadero (GEI) con 23 veces mayor potencial de calentamiento global que el dióxido de carbono y un tiempo de vida media de 10 años en la atmósfera (Solomon et al., 2007). La producción de metano entérico constituye una pérdida energética para el rumiante que representa entre el 2 y el $12 \%$ de la energía bruta consumida (Johnson y Johnson, 1995). Es por esto que algunos autores han propuesto que para la disminución de las emisiones de metano entérico puede ser una estrategia el aumento de la productividad animal (al disminuir las pérdidas energéticas) disminuyendo al mismo tiempo el impacto ambiental (al disminuir las emisiones de metano a la atmósfera) (Neely et al., 2009).

En la literatura se reportan múltiples revisiones sobre la producción de metano por los rumiantes (Johnson y Johnson, 1995; Boadi et al., 2004; Martin et al., 2009; Patra y Saxena et al., 2010), algunas de ellas orientadas a determinar estrategias de alimentación que permitan reducir las emisiones de metano reconociendo factores del animal (tasa de pasaje, consumo residual), de la dieta (composición, relación forraje: concentrado), inclusión de aditivos (ácidos grasos, taninos purificados) y modificadores de las poblaciones ruminales (ionóforos, vacunas). Sin embargo, ninguna de éstas ha abordado el tema desde el punto de vista del pastoreo a pesar de que un $35 \%$ de la producción de metano entérico proviene de sistemas pastoriles (Niggli et al., 2009).

Debido a las limitaciones para determinar las emisiones de metano entérico en rumiantes en pastoreo, aspectos asociados a la especie vegetal, su madurez y la oferta de forraje no han sido consideradas de manera integral. Esta revisión integró las publicaciones en estas áreas identificando los factores que influyen en la producción de metano entérico, así como áreas en las cuales se requiere investigación con el fin de contribuir y reconocer estrategias de manejo que permitan mitigar las emisiones de metano en los sistemas pastoriles. En esta revisión se ha privilegiado la expresión de la producción de metano en términos de la materia seca (MS) consumida en los ensayos in vivo y en referencia a la materia orgánica degradada en los ensayos in vitro, cundo se haga de otra manera se hará explícito en el texto. Es importante considerar que las unidades en las que se expresa la producción de metano permite enfatizar en características fisiológicas, composicionales o productivas que permiten describir la producción de metano debiendo tener precaución al comparar resultados de diferentes experimentos. Sin embargo, es importante resaltar que en los procesos de mitigación la forma de expresión debe propender a mantener la eficiencia productiva del animal además de disminuir el impacto medio-ambiental (Waghorn y Hegarty, 2011).

\section{FORMACIÓNDE METANO}

El metano entérico es un producto de la fermentación anaerobia resultado de un complejo sistema simbiótico entre diferentes grupos microbiales presentes a lo largo del tracto digestivo del rumiante, fundamentalmente en el rumen (Van Soest, 1994). Los microorganismos del rumen metabolizan los carbohidratos de la dieta convirtiéndolos principalmente en ácidos grasos volátiles como acetato, propionato $\mathrm{y}$ butirato. En el proceso de síntesis de acetato y butirato se producen moléculas de hidrógeno las cuales deben ser removidas para mantener la eficiencia energética durante los procesos de fermentación anaerobia (Beauchemin et al., 2008). Las principales vías de remoción de hidrógeno se hacen a través de la biohidrogenación de ácidos grasos insaturados, la formación de ácido propiónico y de metano (Van Soest, 1994). La formación de metano por un grupo de microorganismos metanogénicos (Archaea) es la vía mas importante de remoción de hidrogeno. Los Archaea más destacados en el rumen son Methanobrevibacter 


\section{METANO ENTÉRICO EN SISTEMASPASTORILES}

ruminantium, Methanobacterium formicicum y Methanomicrobium mobile, que reducen el dióxido de carbono usando el hidrógeno como fuente de energía (Attwood y McSweeney, 2008).

\section{ESPECIES FORRAJERAS Y LA PRODUCCIÓNDEMETANO ENTÉRICO}

La literatura sugiere que se pueden encontrar diferencias en la emisión de metano entérico cuando se evalúan diferentes especies forrajeras como fuente de alimento. Por ejemplo, Mieres et al. (2002) encontraron que el uso de pasturas nativas producía mayores cantidades de metano por unidad de MS consumida que una pastura mejorada o introducida, resultado de sus características composicionales. Debido a la gran diversidad de especies forrajeras estudiadas en esta revisión se clasifican los trabajos publicados en tres grandes grupos donde se compararon gramíneas $\mathrm{C} 3$ y C4, variedades dentro de un mismo forraje y gramíneas y leguminosas.

\section{GRAMÍNEAS C3 VSC4}

Las plantas $\mathrm{C} 3$ y $\mathrm{C} 4$ se diferencian en que su primer producto de la fotosíntesis es un compuesto de tres (ácido 3 fosfoglicérico) o cuatro (ácido oxalacético) carbonos, respectivamente (Salisbury y Ross, 2000). En general las gramíneas forrajeras C3 debido a sus diferencias fisiológicas están compuestas por una mayor proporción de tejidos de alta digestibilidad respecto a las C4 (Wilson, 1993). Las primeras investigaciones que compararon la producción de metano en especies $\mathrm{C} 3$ y C4 fueron realizadas por Margan et al. (1988) quienes concluyeron que ovejas alimentadas con especies C4 (Digitaria decumbens y Setaria sphacelata) produjeron $14,3 \%$ más metano por unidad de energía digestible consumida que aquéllas alimentadas con especies C3 (ryegrass perenne (Lolium perenne) y tré- bol blanco (Trifolium repens)). El consumo de especies $\mathrm{C} 4$ en comparación con las $\mathrm{C} 3$ se han relacionado con una menor producción de leche y/o carne, lo cual resulta en una mayor emisión de metano por unidad de producto (i.e., intensidad de emisión) (Waghorn y Hegarty, 2011).

Algunos autores sugieren que la mayor producción de metano asociados con la alimentación de forrajes $\mathrm{C} 4$ puede deberse a un mayor contenido de carbohidratos estructurales y lignina (Kamra et al., 2010), menor consumo y tasa de pasaje más lenta (McAllister et al., 1996). Asimismo, las ecuaciones desarrolladas por Moe y Tyrrell (1979) evaluando 404 ensayos desarrollados con ganado de leche sugieren que dietas a base de carbohidratos estructurales producen mayores proporciones de metano por unidad degradada que aquéllas donde prevalecen los carbohidratos no estructurales. Otros autores sugieren que en animales alimentados con forrajes tropicales se produce más metano $(>\mathbf{8 \%})$ por unidad de energía bruta consumida que con aquéllos de zonas templadas (7\%) debido a una mayor proporción de carbohidratos estructurales (Clark et al., 2005).

Otras investigaciones sugieren que no hay efecto del tipo de especie $\mathrm{C} 3$ o $\mathrm{C} 4$ sobre las emisiones de metano (Klevenhusen et al., 2009). Además en forrajes tropicales y templados no se ha encontrado una clara asociación entre los componentes de la dieta y la producción de metano (Clark et al., 2005). Algunos autores sugieren que las diferencias en la variación en la producción de metano entre especies puede relacionarse en una mayor medida a diferencias en el nivel de consumo (Muetzel et al., 2009; Sun et al., 2011a), tasas de degradación y paso diferencial entre especies forrajeras (Pinares-Patiño et al., 2007a) que a la composición de la dieta (Sun et al., 2011b) y la especie animal (Primavesi et al., 2004). Es por esto que hay que ser cuidadoso al generalizar estos resultados y debe hacerse una mayor investigación en torno a la produc- 
ción de metano en especies C3 y C4 (Kurihara et al., 1999; McCrabb y Hunter, 1999; Clark et al., 2005).

\section{ESPECIES Y VARIEDADES}

Muy pocos trabajos se han enfocado en reconocer las diferencias en las emisiones de metano entre diferentes variedades de una misma especie. Es posible que las diferencias en la calidad nutricional de cada variedad dentro de una especie puedan modificar las emisiones de metano. En estudios in vitro donde se incluyó ryegrasses con $12 \%$ más de azúcares solubles mostró una reducción de metano en un 10\% (Lovett et al., 2005). Sin embargo, otros trabajos in vitro no han reportado diferencia entre variedades altas y bajas en carbohidratos solubles (Lovett et al., 2004; Zeleke et al., 2006; Navarro-Villa et al., 2011). Misselbrook et al. (2010) observaron in vivo que variedades de ryegrass con mayores contenidos de carbohidratos solubles presentaron una menor producción de metano $(24 \%)$.

Primavesi et al. (2003) compararon las emisiones de metano provenientes de animales en pastoreo que recibieron suplementación de caña de azúcar con mayor y menor contenido de fibra en detergente neutro, reportando un $30 \%$ menos metano por unidad de peso vivo en aquéllas dietas más ricas en carbohidratos no estructurales. Además estos autores sugirieron que cuando se incluye un alimento balanceado en un $40 \%$ de la dieta, con bajo contenido de fibra, produce menos metano por unidad de producto (carne o leche).

Una mayor concentración de proteína cruda ha sido asociada con la disminución en las emisiones de metano. En ensayos in vitro donde se comparó ryegrass perenne con $75 \%$ mas proteína cruda se reportó $14 \%$ menos metano por unidad de materia orgánica degradada (Lovett et al., 2004). Resultados similares fuero reportados por Navarro-Villa et al. (2011) quienes observaron una disminución de 7\% en la emisión de metano por unidad de MS incubada en ryegrass perenne con $3 \%$ más de proteína cruda.

La poca información relacionada con emisiones de metano asociadas a diferencias en variedades de una misma especie sugeriría que el uso de variedades bajas en fibra y ricas en azúcares y proteína cruda podría ser una opción viable para reducir las emisiones de metano por unidad de producto (carne o leche) al aumentar la productividad animal. En este sentido Ellis et al. (2012) sugiere que la alimentación de forrajes con mayor concentración de azúcares o proteína disminuye hasta en un $13 \%$ la producción de metano por unidad de producto.

\section{GRAMÍNEAS VS LEGUMINOSAS}

En estudios in vitro la inclusión de leguminosas tropicales bajas en taninos condensados ha presentado un aumento en las emisiones de metano por unidad de materia orgánica fermentada asociado a una mayor degradabilidad de nutrientes (Hess et al., 2003; Tiemann et al., 2008a). Hess et al. (2003), evaluaron la inclusión de una leguminosa baja en taninos (Arachis pintoi) en proporciones crecientes $(33,66$ y $100 \%)$ en una dieta base de gramínea (Brachiaria humidicola) y encontraron una relación lineal creciente entre la inclusión de leguminosa y la producción de metano por unidad de FDN fermentada. Igualmente Tiemann et al. (2008a) sugieren un aumento del $35 \%$ en la producción de metano por unidad de FDN fermentada cuando se incluye una leguminosa baja en taninos (Vignia ungiculata) en una dieta a base de gramínea (Brachiaria humidícola) en proporción 1:2, comparando con la gramínea pura.

Estudios in vitro con leguminosas y gramíneas de zonas templadas muestran resultados completamente diferentes a los estudios con forrajes tropicales. NavarroVilla et al. (2011) reportaron una disminución de $7 \%$ en la producción de metano 


\section{METANO ENTÉRICOEN SISTEMAS PASTORILES}

por unidad de MS incubada al comparar trébol respecto a ryegrass perenne. En estudios in vivo McCaughey et al. (1999) observaron una reducción de un $25 \%$ de las emisiones de metano por energía bruta ingerida cuando se comparó una dieta de gramínea (Bromus biebersteinii) con una asociación de gramínea: leguminosa (Bromus biebersteinii: Medicago sativa). Otros trabajos donde se ha sustituido la gramínea Bromus bieberteinii por $\mathrm{Me}$ dicago sativa han presentado disminuciones del $20 \%$ en las emisiones de metano por unidad de MS consumida (Chavez et al., 2006), razón por la cual Martin et al. (2009) sugirieron que la inclusión de Medicago sativa promueve una mayor tasa de pasaje y debido a sus altos contenidos de malato en las hojas, el cual es un intermediario en la vía de producción de propionato, podría disminuir la producción de metano entérico al aumentar la relación propionato: acetato en el rumen. De Klein et al. (2008) sugirieron que la inclusión de leguminosas en la dieta podría disminuir la producción de metano por unidad de MS consumida hasta en un $53 \%$. Esto se ha relacionado con un menor contenido de fibra en las dietas con leguminosas, una reducción en el $\mathrm{pH}$, aumento del amonio, ácidos grasos volátiles y relación propionato: acetato en el rumen, mayor tasa de pasaje y la presencia de algunos metabolitos secundarios (Mieres et al.,2002; Williams et al., 2005; Waghorn y Clark, 2006; Waghorn, 2008; Beauchemin et al., 2008; Eckard et al., 2010).

La inclusión de leguminosas de zona templada en dietas de gramíneas tropicales ha mostrado resultados similares a lo reportado para dietas de zonas templadas. Hunter (2007) compararon la producción de metano en novillos Brahman que recibieron una dieta de gramíneas tropicales como Dichanthium aristatum y Chloris gayana, frente a aquellos que consumieron alfalfa (Medicago sativa), y reportaron emisiones de metano por unidad de ganancia de peso 4 veces más altas en animales alimentados con gramíneas comparado con los de leguminosa.

No todos los estudios con leguminosas de zonas templadas han mostrado una disminución en las emisiones de metano. Por ejemplo estudios reportados por McCaughey et al. (1999); Waghorn et al. (2002); De Klein et al. (2008) y O'Mara et al. (2008) han mostrado aumentos en las emisiones de metano por unidad de MS consumida al incluir una leguminosa en la dieta, debido a un mayor consumo voluntario, aumento en digestibilidad de la dieta y modificación de los patrones de fermentación asociados a la inclusión de la leguminosa. Carulla et al. (2005) reportaron un aumento en las emisiones de metano por unidad de MS consumida asociadas a la inclusión de ensilaje de trébol o alfalfa en una dieta de ryegrass perenne. Sin embargo, otros trabajos no han reportado diferencias entre gramíneas y leguminosas (Beever et al., 1985; Van Dorland, 2007). Por último, algunos autores han sugerido que la respuesta puede depender de las especies asociadas. Por ejemplo, Knight et al. (2007) reportaron $23 \%$ menos metano por unidad de MS ingerida por corderos que consumieron trébol blanco respecto a los alimentados con ryegrass perenne, mientras que observaron una mayor emisión (21\%) en corderos que consumieron Trifolium ambiguum en comparación con ryegrass perenne.

A pesar que no exista un consenso entre el efecto que puede producir animales alimentados con gramíneas $\mathrm{C} 3$ y $\mathrm{C} 4$ o con diferentes niveles de inclusión de leguminosas en zonas templadas y cálidas sobre la producción de metano entérico, si se han observado diferencias entre leguminosas y su efecto sobre la producción de metano al asociarlas con algunos compuestos secundarios encontrados en ellas como son los taninos y las saponinas. La concentración y tipo de estos compuestos varía entre especies, procedencia, estado fisiológico de la planta, año y época de cosecha lo cual dificulta su estudio. A continuación se 
discuten los efectos de los taninos y las saponinas presentes en las especies forrajeras.

Taninos - Los taninos son polifenoles capaces de ligarse a compuestos de la dieta (proteínas, carbohidratos) (Cortés et al., 2009; Patra y Saxena, 2010; Jakmola et al., 2010). Esto podría disminuir la degradabilidad de algunos nutrientes de la dieta, inhibir la actividad enzimática, disminuir las poblaciones de protozoarios o de bacterias celulolíticas y en consecuencia disminuir la producción de metano (McSweeney et al., 2001; Tavendale et al., 2005; Johnson et al., 2007; Tiemann et al., 2008a; Hess et al., 2008; Jakmola et al., 2010).

El efecto de los taninos en las leguminosas sobre la producción de metano aparentemente es muy variable y depende del tipo de taninos y su concentración en la planta. Se ha reportado in vitro una mayor disminución en las emisiones de metano cuando se incluyen taninos condensados frente a los hidrolizables, debido a una mayor reducción en las poblaciones de microrganismos metanogénicos y de protozoarios (Bhatta et al., 2009). Estos autores, en estudios in vitro, han mostrado una relación inversa y lineal entre la concentración de taninos condensados y la producción de metano. Sin embargo, aunque algunos estudios in vivo en donde se incluyen taninos condensados en la dieta muestran una disminución en la metanogénesis en ovejas (e.g., Carulla et al., 2005) otros no han reportado ningún efecto (e.g., Beauchemin et al., 2007).

La presencia de leguminosas taníferas en dietas de bovinos y ovinos se ha relacionado con disminución en las emisiones de metano (Woodward et al., 2001; PinaresPatiño et al., 2003a). Diferentes estudios con leguminosas de zonas templadas reportan una disminución entre un 12 y un $60 \%$ sobre la producción de metano como proporción de la energía bruta consumida cuando se incluye en la dieta una leguminosa con taninos condensados (Woodward et al.,
2001; Pinares-Patiño et al., 2003a; Waghorn et al., 2002). Por ejemplo, la inclusión de Lotus corniculatus en la dieta de bovinos fue asociada con 16-25\% menor emisión de metano por unidad de MS consumida o por unidad de producto animal en bovinos y ovinos (Woodward et al., 2001; Waghorn et al., 2002). Estos efectos también han sido corroborados en estudios in vitro e in vivo con leguminosas tropicales. Por ejemplo, en estudios in vivo con ovinos se ha evaluado la inclusión de hojas de leguminosas arbustivas (Flemingia macrophylla y Calliandra calothyrsus) que contienen taninos condensados, y se encontraron disminuciones en las emisiones de metano entre 20 y $30 \%$ explicados principalmente por una reducción en la degradabilidad de la materia seca (Tiemann et al., 2008b). De tal forma, Waghorn (2008) y Bouchard (2011) sugieren que la respuesta del animal al consumo de taninos es dependiente de la concentración de éstos en la dieta, reportándose efectos benéficos cuando la concentración varía entre 2 y $4 \%$ y negativos con inclusiones mayores al $6 \%$.

Finalmente, algunos autores han sugerido que taninos condensados de bajo peso molecular, según su estructura química, pueden formar complejos más estables con las enzimas microbiales disminuyendo su actividad, mientras que los de alto peso tienen una menor capacidad de afectar las poblaciones metanogénicas (Field et al., 1989). Otros autores han propuesto que taninos de mayor peso molecular tienen mayor capacidad de reaccionar con los componentes de la dieta (Huang et al., 2011). Waghorn (2008) sugiere que el peso molecular de los taninos no es el único factor que influye en su actividad. La concentración del tanino en la planta y su actividad biológica (Tiemann et al., 2008a; Theodoridou et al., 2011) varían dependiendo de las condiciones ambientales (fertilidad del suelo, lluvias o sequia)(Chesson y Forsberg, 1997). Adicionalmente, algunos 


\section{METANO ENTÉRICOEN SISTEMAS PASTORILES}

autores sugieren que los microorganismos ruminales pueden desarrollar mecanismos de adaptación evitando el efecto de los taninos (Smith et al., 2005). Por lo tanto, el efecto de estos compuestos en la producción de metano depende de gran variedad de factores que deben ser considerados en condiciones específicas de cada especie de leguminosa en un ambiente determinado.

Saponinas - Algunas especies de leguminosas contienen saponinas como es el caso de la alfalfa (Medicago sativa). Las saponinas son glucósidos de alto peso molecular que disminuyen la tasa de producción de metano (Guo et al., 2008; Patra y Saxena, 2010). También interactúan con el colesterol presente en la membrana de los protozoarios causando su lisis (Hess et al., 2003, Jakmola et al., 2010). En varios trabajos la defaunación se ha asociado con la reducción entre un 15 y $40 \%$ de la producción de metano (Abreu et al., 2004; McAllister y Newbold, 2008). Se ha sugerido que este efecto se debe a la disminución en la trasferencia de hidrógenos desde los protozoarios a los microrganismos metanogénicos asociados a la superficie de éstos y reducción en la digestión de la fibra (Hegarty, 1999; Machmüller et al., 2003; Buddle et al., 2011), además de un aumento en la concentración de ácido propiónico (Guo et al., 2008; Wang et al., 2009) y de biomasa microbial (Makkar et al., 1996).

No todas las saponinas tienen el mismo efecto (Hess et al, 2003; Beauchemin et al., 2008; Guo et al., 2008). Algunas investigaciones han sugerido que no existe una relación directa entre la adición de saponinas, la defaunación y la reducción en la producción de metano (Hess et al., 2003; Goel et al., 2008). En dietas basadas en forrajes los intentos de defaunación son menos efectivos que en dietas completamente mezcladas (Hegarty, 1999; Goel et al., 2008; Bird et $a l ., 2008)$. También, se ha sugerido que las poblaciones microbiales se adaptan a la presencia de estos compuestos y los degradan por lo cual su efecto no sería permanen- te en el tiempo (Hess et al., 2003).

De la información revisada no es posible concluir si la inclusión de una leguminosa en la dieta disminuye las emisiones de metano. Existen diferencias importantes entre leguminosas como su composición nutricional, su digestibilidad y presencia o no de metabolitos secundarios (Tavendale et $a l ., 2005)$ que sugieren que cada leguminosa debería considerarse de manera individual. Para aquellas leguminosas más comúnmente usadas en zonas templadas como son la alfalfa y el trébol blanco la mayoría de los resultados sugieren que reducen las emisiones de metano por unidad de MS consumida debido a un mayor consumo de alimento y aumento en las tasas de paso. En estudios donde no se presentó una disminución de metano con el uso de leguminosas como el caso de Carulla et al. (2005) los consumos no se modificaron posiblemente explicando una respuesta diferencial con otros estudios; adicionalmente, estas leguminosas estaban ensiladas. Por otro lado, las leguminosas tropicales incluyen una gran diversidad de géneros y especies lo cual hace difícil concluir sobre las mismas. Los pocos trabajos que se han realizado con ellas muestran un aumento en la producción de metano cuando éstas no contienen factores antinutricionales como los taninos (Hess et al., 2006). Este aumento se debería a una mejoría en las condiciones de fermentación. El impacto de estos hallazgos en animales en pastoreo debe ser investigado ya que es probable que bajo estas circunstancias aumente el consumo voluntario y las tasas de paso, reduciendo la producción de metano por unidad de materia seca ingerida o por unidad de producto animal.

\section{MADUREZDELFORRAJE}

En estudios in vitro la respuesta de la madurez del forraje sobre la producción de metano ha sido contradictoria. Navarro-Villa et al. (2011) y Purcell et al. (2012) sugi- 
rieron que una mayor madurez en ryegrass perenne aumenta las emisiones de metano por unidad de materia seca incubada. Sin embargo para la misma gramínea, Purcell et al. (2011) reportan una disminución en las emisiones de metano por unidad de materia seca incubada cuando aumenta la madurez del forraje. En la literatura pocos trabajos han evaluado el efecto de la madurez de las leguminosas sobre la producción de metano in vitro. Estudios in vivo con pasturas de zonas templadas como L. perenne, Phleum pratense y $T$. repens reportan que la mayor madurez del forraje se relaciona con un aumento, aunque no significativo, en la producción de metano por unidad de MS consumida (Beever et al., 1985; Cammel et al., 1986; Pinares-Patiño et al., 2003b; Molano y Clark, 2008). Otros autores sugieren que a pesar de que no se encuentren diferencias significativas en las producción de metano por unidad de MS consumida entre forrajes jóvenes y maduros, podría ser menor la producción de metano por unidad de producto animal en forrajes tiernos debido a una aumento de la productividad animal(Clark et al., 2005).

El efecto de la madurez sobre las emisiones de metano aparentemente no es claro. Sin embargo, en sus revisiones Beauchemin et al. (2008) y Martin et al. (2009) sugieren que la menor producción de metano en forrajes más tiernos podría estar asociada a una mayor concentración de ácido linolénico, azúcares solubles, y una mayor tasa de paso. Otras explicaciones podrían estar asociadas a las mayores digestibilidades de los forrajes tiernos y el cambio que esta mayor digestibilidad genera en el pH ruminal. A continuación se discutirán estos aspectos en detalle.

Lípidos - Beauchemin et al. (2008) sugirieron que los ácidos grasos insaturados tienen la capacidad de capturar hidrógeno debido a los procesos de biohidrogenación ruminal, sin embargo su eficiencia es mucho menor que la formación de metano (Johnson y Johnson, 1995). Estos autores proponen que un mayor grado de insaturación tendría mayor capacidad de captura de hidrógeno. A pesar de esto, las concentraciones de lípidos en los forrajes son bajas (2-5\%) (Swainson, 2011) y de éstos cerca del 50\% son ácidos grasos (Palmquist y Jenkins, 2003) de los cuales el 50-60\% son insaturados (Aguilar et al., 2009). Además, los forrajes tiernos normalmente contienen mayores concentraciones de lípidos, con mayores proporciones de ácidos grasos insaturados (particularmente ácido linolénico) que los maduros (Sun et al., 2010; Aguilar et al., 2009), sugiriendo que forrajes más jóvenes tendrían mayor potencial de reducción de metano.

Carbohidratos solubles - Los forrajes maduros tienen una mayor concentración de carbohidratos estructurales (Sun et al., 2010) que ha sido relacionada directamente con una mayor producción de metano (Pinares-Patiño et al., 2007b; Jayanegara et al., 2009). Estos cambios implicarían que en forrajes tiernos con mayores concentraciones de carbohidratos solubles favorecen las rutas de degradación de los carbohidratos hacia propionato (Murphy et al., 1982). Aunque la literatura ha evi-denciado que al aumentar los almidones se favorecen estas rutas (Van Soest, 1994) esto ha sido menos evidente para los carbohidratos solubles en los pastos (Purcell et al., 2012) y en algunos casos opuesto (Navarro-Villa et al., 2011). Varios autores no han encontrado cambios en el patrón de AGV asociados a la madurez o concentración de azúcares solubles in vitro (Hall et al., 1998; Taweel et al., 2005) aunque otros han reportado mayores proporciones de propionato para forrajes ricos en carbohidratos solubles (Purcell et al., 2011).

Tasas de paso- Pinares-Patiño et al. (2003c y 2007a) han relacionado inversamente la tasa de paso con la producción de metano debido a una mayor extensión en la degradabilidad de la fibra resultando en una mayor producción de metano. Adicionalmente, los diferentes carbohi- 


\section{METANO ENTÉRICO EN SISTEMASPASTORILES}

dratos tienen rutas de degradación compartidas por lo cual los cambios en la composición del forraje debido a la madurez difícilmente explicarían las variaciones en la producción de metano. Los productos finales de la degradación de los carbohidratos están más asociados a los microorganismos que los degradan, los cuales dependen del ambiente ruminal (tasas de paso, $\mathrm{pH}$ ) (Murphy et al., 1982). Los cambios en las poblaciones microbiales ruminales debido a mayores tasas de paso en estos estudios no han sido caracterizadas. Sin embargo, a mayores tasas de paso se disminuyen las proporciones de hongos ruminales y protozoarios (Dehority y Orpin, 1997) además de la digestibilidad de la materia orgánica (Alwash y Thomas, 1971). El rol de los protozoarios en la producción de metano ha sido ampliamente documentado (Williams y Coleman, 1997; Hegarty, 1999) y en menor grado el de los hongos (Orpin y Joblin, 1997), aunado a esto Pinares-Patiño et al. (2003c) reportan que el contenido de materia orgánica en el rumen está directamente relacionado con la producción de metano. Esto sugeriría que en las dietas con forrajes jóvenes las poblaciones de hongos y protozoarios son menores, además se reduciría la digestibilidad de la materia orgánica resultando en una disminución de la producción de metano. Esta relación debería ser verificada en estudios donde se evalúe la producción de metano y los cambios en las poblaciones microbiales debido a los cambios de la madurez del forraje y las tasas de pasaje.

pH ruminal- Forrajes jóvenes tienen mayor digestibilidad que aquellos maduros (Navarro-Villa et al., 2011), esta diferencia implicaría una mayor concentración de AGV en el fluido ruminal y por lo tanto un menor $\mathrm{pH}$. Algunos trabajos in vitro han reportado menores valores de $\mathrm{pH}$ cuando se incuban pasturas tiernas (Purcell et al., 2012; Navarro-Villa et al., 2011), lo que sugeriría también cambios en las poblaciones mi- crobiales asociados a menores valores $\mathrm{pH}$ (Kaufman et al., 1980). Los pH inferiores a 6,2 son menos favorables para el crecimiento de micro-organismos fibrolíticos (Van Soest, 1994) que normalmente producen mayores proporciones de acetato en el fluído y por lo tanto mayores cantidades de metano por unidad de materia orgánica degradada (Pinares-Patiño et al., 2007a). El impacto sobre las poblaciones microbiales dependería de las diferencias entre la madurez del forraje y el consumo de alimento.

\section{MANEJO DE LA PASTURA}

Existen pocos trabajos referentes a los aspectos asociados al manejo de la pastura (sistema de pastoreo, oferta forrajera, carga animal) y su efecto en la emisión de metano. DeRamus et al. (2003) reportaron que existe una disminución del $22 \%$ en las emisiones de metano por unidad de producto animal cuando se compara un manejo tradicional de la pastura (continuo, sin rotación, sin fertilización) con un manejo intensivo rotacional (con fertilización y resiembra), debido a un aumento en la producción y calidad del forraje reflejándose en una mayor eficiencia animal a través del año. Desafortunadamente, en este ensayo fue difícil separar los aspectos asociados a las diferentes variables consideradas como manejo.

Otros estudios han considerado el efecto de la oferta forrajera sobre la producción de metano. Los estudios han sugerido que al aumentar la disponibilidad de forraje en un $60 \%$, se aumentan las emisiones de metano por unidad animal en un $41 \%$, pero disminuyen por unidad de materia seca consumida en un $23 \%$, posiblemente relacionado con un aumento en la tasa de pasaje (Pinares-Patiño et al., 2003d). Otros trabajos argumentan que una menor cantidad de forraje residual en el anterior pastoreo y por lo tanto una mayor presión de pastoreo disminuye la producción de metano, debido 
a una menor concentración de carbohidratos estructurales en la composición de la pradera (Wims et al., 2010). McCaughey et al. (1997) sugieren que al aumentar la carga de 1,1 a 2,2 animales por hectárea, se disminuyen las emisiones de metano por unidad de materia orgánica fermentada en un $15 \%$, debido a una disminución en la concentración de carbohidratos estructurales de la pradera, lo que disminuye el tiempo de retención del alimento en el rumen. Estos trabajos parecerían contradictorios pues para lograr un menor forraje residual se requiere una menor oferta forrajera por animal. Por lo tanto, el efecto a largo plazo de una mayor oferta (i.e. menor carga animal) podría considerarse negativo debido a una disminución en la calidad de la pastura. Aquí se requerirían investigaciones de largo plazo en pastoreo comparando altas y bajas ofertas de forraje que permitan evidenciar el efecto de dicho manejo sobre las emisiones de metano.

\section{FERTILIZACIÓN}

La emisión de metano por unidad de MS consumida disminuye cuando se incrementa el nivel de fertilización de la pastura (Ellis et al., 2012). Bannink et al. (2009) reportan una disminución del $8 \%$ en la producción de metano cuando se comparó una fertilización de 450 vs $150 \mathrm{~kg}$ de nitrógeno por hectárea año. Sin embargo en pasturas asociadas de gramínea y leguminosa no se presentaron diferencias en las emisiones de metano cuando se aplicó una fertilización nitrogenada (Mbanzamihigo et al., 2002). En estudios in vitro, Lovett et al. (2004) y Navarro-Villa et al. (2011) encontraron una relación lineal negativa entre la aplicación de nitrógeno al suelo y la producción de metano por unidad de materia orgánica degradada, debido posiblemente a una disminución en la degradabilidad de la materia orgánica en pasturas que recibieron niveles crecientes de $\mathrm{N}$ y a mayores concentraciones de nitratos (Leng, 2008).

\section{CONSUMO VOLUNTARIOYEMISIÓN DEMETANO ENTÉRICO}

La literatura reporta que el consumo de MS es el principal factor que influye sobre la producción de $\mathrm{CH}_{4}$ entérico (Buddle et al., 2011). Las primeras investigaciones que evaluaron el efecto del consumo sobre la producción de $\mathrm{CH}_{4}$ encontraron una estrecha relación con la cantidad de alimento y la digestibilidad de la dieta, presentándose una mayor producción de $\mathrm{CH}_{4}$ en dietas de baja digestibilidad a un nivel de consumo de mantenimiento, por el contrario dietas con alta digestibilidad y un consumo tres veces mayor al de mantenimiento disminuyó la producción de $\mathrm{CH}_{4}$ (30\%) (Blaxter y Clapperton, 1965). El aumento en el consumo de MS se relaciona con una mayor tasa de paso y la disminución en la degradabilidad de la dieta, lo que resulta en una menor producción de metano (Pinares-Patiño et al., 2003c).

Investigaciones recientes han sugerido que el aumento en el nivel de consumo en dietas ricas en forrajes disminuye las emisiones de metano por unidad de materia seca consumida (11 a 14\%) (Sun et al., 2011 a; Hammond et al., 2011). Sin embargo, la magnitud en la reducción no es tan grande como aquella reportada por Blaxter and Clapperton (1965) debido probablemente a la poca variabilidad en composición química y digestibilidad de los forrajes evaluados.

La relación entre consumo voluntario y emisión de metano por unidad de consumo sugiere que todas aquellas prácticas asociadas al aumento del consumo de MS de los forrajes serían potencialmente conducentes a una menor producción de metano por unidad de MS ingerida.

\section{CONSIDERACIONESFINALES}

Esta revisión de literatura sugiere que existen diferentes herramientas que permitirían disminuir la producción metano entérico por unidad de materia seca ingerida en los 


\section{METANO ENTÉRICOEN SISTEMAS PASTORILES}

sistemas de pastoreo. Algunas de éstas, asociadas a la calidad del forraje que está a su vez determinada por aspectos asociados a la especie, la variedad, madurez, presencia de compuestos secundarios y manejo de la pastura. En la mayoría de estudios donde estas herramientas fueron evaluadas muestran que la disminución de metano estuvo asociada a un aumento en el consumo voluntario y las tasas de paso.

Es necesario evaluar con mayor interés

\section{BIBLIOGRAFÍA}

Abreu, A., Carulla, J.E., Lascano, C.E., Diaz, T.E., Kreuzer, M. and Hess, H.D. 2004. Effects of Sapindus saponaria fruits on ruminal fermentation and duodenal nitrogen flow of sheep fed a tropical grass diet with and without legume. J Anim Sci, 82: 1392-1400.

Aguilar, O., Moreno, B., Cárdenas, E., Pabón, M. and Carulla, J. 2009. Efecto del consumo de kikuyo (Pennisetum calndestinum) o ryegrass (Lolium spp.) sobre la concentración de ácido linoléico conjugado y el perfil de ácidos grasos de la grasa láctea. Lives Res Rural Develop, 21: 49.

Alwash, A.H. and Thomas, P.C. 1971. Effect of the physical form of the diet and the level feeding on the digestion of died grass by sheep. J Sci Food Agr, 22: 611- 615.

Attwood, G. and McSweeney, C. 2008. Methanogen genomics to discover targets for methane mitigation technologies and options for alternative $\mathrm{H}_{2}$ utilization in the rumen. Aust J Exp Agri, 48: 28-37.

Bannink, A., Smits, M.C.J., Kebreab, E., Mills, J.A.N., Ellis, J.L., Klop, A., France, J. and Dijkstra, J. 2009. Simulating the effects of grasslands management and grass ensiling on methane emissions from lactating cows. J Agr Sci, 148: 55-72.

Beauchemin, K.A., Kreuzer, M., O'mara, F. and McAllister, T.A. 2008. Nutritional management for enteric methane abatement: a review. Aust J Exp Agri, 48: 21-27.

Beauchemin, K.A., McGinn, S.M., Martinez, T.F. and McAllister, T.A. 2007. Use of condensed tannin extract from quebracho trees to reduce las emisiones de metano en animales en pastoreo, especialmente en condiciones tropicales, pues existe un limitado acervo científico en este campo. Además es importante reconocer que para desarrollar e implementar estrategias de mitigación en los sistemas pastoriles es necesario considerar la integridad de los GEI procedentes del sector ganadero y evaluar los diferentes escenarios bajo un enfoque global (huella de carbono o ciclo de vida).

methane emissions from cattle. J Anim Sci, 85: 1990-1996.

Beever, D.E., Thomson, D.J., Ulyatt, M.J., Cammell, S.B. and Spooner, M.C. 1985. The digestion of fresh perennial ryegrass (Lolium perenne $\mathrm{L}$. cv. Melle) and white clover (Trifolium repens $L$. cv. Blanca) by growing cattle fed indoors. Brit J Nutr, 54: 763-775.

Bhatta, R., Uyeno, Y., Tajima, K., Takenaka, A., Yabumoto, Y., Nonaka, I., Enishi, O. and Kurihara, M. 2009. Difference in the nature of tannins on in vitro ruminal methane and volatile fatty acid production and on methanogenic archea and protozoal populations. J Dairy Sci, 92: 55125522.

Bird, S.H., Hegarty, R.S. and Woodgate, R. 2008. Persistence of defaunation effects on digestion and methane production in ewes. Aust $J$ Exp Agri, 48: 152-155.

Blaxter, K.L. and Clapperton, J.L. 1965. Prediction of the amount of methane produced by ruminants. Brit J Nutr, 19: 511-522.

Boadi, D., Benchaar, C., Chiquette, J. and Massé, D. 2004. Mitigation strategies to reduce enteric methane emissions from dairy cows: Update review. Can J Anim Sci, 84: 319-335.

Bouchard, K. 2011. Methane emissions and rumen microbial changes in steers fed condensed tannin containing diets under western Canadian conditions. Master of Science Thesis. Department of Animal Science. University of Manitoba. Winnipeg, MB. Canadá.

Buddle, B.M., Denis, M., Attwood, G.T., Altermann, E., Janssen, P.H., Ronimus, R.S., Pinares-Patiño, C.S., Muetzel, S. and Wedlock, D.N. 2011. 


\section{VARGAS, CÁRDENAS, PABÓNY CARULLA}

Review: Strategies to reduce methane emissions from farmed ruminants grazing on pastures. The Vet J, 188: 11-17.

Cammel, S.B., Thomson, D.J., Beever, D.E., Haines, M.J., Dhanoa, M.S. and Spooner, M.C. 1986. The efficiency of energy utilization in growing cattle consuming fresh perennial ryegrass (Lolium perenne cv. Melle) or white clover (Trifolium repens cv. Blanca). Brit J Nutr, 55: 669-680.

Carulla, J.E., Kreuzer, M., Machmüller, A. and Hess, H.D. 2005. Supplementation of Acacia mearnsii tannin decreases methanogenesis and urinary nitrogen in forage-fed sheep. Aust J Agr Res, 56: 961-970.

Chavez, A.V., Thompson, L.C., Iwaasa, A.D., Scott, S.L., Olson, M.E., Benchaar, C., Veira, D.M. and McAllister, T.A. 2006. Effect of pasture type (alfalfa vs grass) on methane and carbon dioxide production by yearling beef heifers. Can J Anim Sci, 86: 409-418.

Chesson, A. and Forsberg, C.W. 1997. Polysaccharide degradation by rumen microorganisms. In: The rumen microbial ecosystem. Hobson, P.N. and Stewart, C.S. (Eds.). Blackie Academic \& Professional. London, UK. pp. 329404.

Clark, H., Pinares-Patiño, C. and De Klein, C. 2005. Methane and nitrous oxide emissions from grazed grasslands. In: McGilloway, D.A. Grassland: A global resource. Academic Publishers. Wageningen, The Netherlands. pp. 279-293.

Cortés, J.E., Moreno, B., Pabón, M.L., Avila, P., Kreuzer, M., Hess, H.D. and Carulla, J.E. 2009. Effects of purified condensed tannins extracted from Calliandra, Flemingia and Leucaena on ruminal and posruminal degradation of soybean meal as estimated in vitro. Anim Feed Sci Technol, 151: 194-204.

Dehority, B.A. and Orpin, C.G. 1997. Development of, and natural fluctuations in, rumen microbial populations. In: The rumen microbial ecosystem. Hobson, P.N. and Stewart, C.S. (Eds.). Blackie Academic \& Professional. London, UK. pp. 204218.

De Klein, C.A.M., Pinares-Patiño, C. and Waghorn, G.C. 2008. Greenhouse gas emissions. In: Environmental impacts of pasture-based farming. McDowell RW. CAB International. London, UK. pp. 1-32.
DeRamus, H.A., Clement, T.C., Giampola, D.D. and Dickison, P.C. 2003. Methane emissions of beef cattle on forage: Efficiency of grazing management system. J Environ Qual, 32: 269-277.

Eckard, R.J., Grainger, C. and De Klein, C.A.M. 2010. Options for the abatement of methane and nitrous oxide from ruminant production: $A$ review. Livest Sci, 130: 47-56.

Ellis, J.L., Dijkstra, J., France, J., Parson, A.J., Edwards, G.R., Rasmussen, S., Kebreab, E. and Bannink, A. 2012. Effect of high-sugar grasses on methane emissions simulated using a dymanic model. J Dairy Sci, 95: 272-285.

Field J.A., Kortekaas S, y Lettinga G. 1989. The tannin theory of methanogenic toxicity. Biol Wastes, 29: 241-262.

Goel, G., Makkar, H.P.D. and Becker, K. 2008. Effects of Sesbania sesban and Carduus pycnocephalus leaves and Fenugreek (Trigonella foenum-graecum L.) seed and their extracts on partitioning of nutrients from roughage and concentrate based feeds to methane. Anim Feed Sci Technol, 147: 72-89.

Guo, Y.Q., Liu, J.X., Lu, Y., Zhu, W.Y., Denman, S.E. and McSweeney, C.S. 2008. Effect of tea saponin on methanogenesis, microbial community structure and expression of mcrA gene, in cultures of rumen micro-organisms. Lett Appl Microbiol, 47: 421-426.

Hall, M.B., Pell, A.N. and Chase, L.E. 1998. Characteristics of neutral detergent-soluble fiber fermentation by mixed ruminal microbes. Anim Feed Sci Technol, 70: 23-39.

Hammond, K.J., Hoskin, S.O., Burke, J.L., Waghorn, G.C., Koolaard, J.P. and Muetzel, S. 2011. Effects of feeding fresh with clover (Trifolium repens) or perennial ryegrass (Lolium perenne) on enteric methane emissions from sheep. Anim Feed Sci Tech, 166-167: 398-404.

Hegarty, R.S. 1999. Reducing rumen methane emissions through elimination of rumen protozoa. Aust J Agri Res, 50: 1321-1327.

Hess, H.D., Mera, M.L., Tiemann, T.T., Lascano, C.E. and Kreuzer, M. 2008. In vitro assessment of the suitability of replacing the low-tannin legume Vigna unguiculata with the tanniniferous legumes Leucaena leucocephala, Flemingia macrophylla or Calliandra calothyrsus in a tropical grass diet. Anim Feed Sci Technol, 147: 105-115. 


\section{METANO ENTÉRICOEN SISTEMAS PASTORILES}

Hess, H.D., Tiemann, T.T., Noto, F., Carulla, J.E. and Kreuzer, M. 2006. Strategic use of tannins as means to limit methane emission from ruminant livestock. Int Cong Series, 1293: 164-167.

Hess, H.D., Monsalve, L.M., Lascano, C.E., Carulla, J.E., Díaz, T.E. and Kreuzer M. 2003. Supplementation of a tropical grass diet with forage legumes and Sapindus saponaria fruits: effects on in vitro ruminal nitrogen turnover and methanogenesis. Aust J Agri Res, 54: 703-713.

Huang, X.D., Liang, J.B., Tan, H.Y., Yahya, R., Khamseekhiew, B. and Ho, Y.W. 2011. Molecular weight and protein binding affinity of Leucaena condensed tannins and their effects on in vitro fermentation parameters. Anim Feed Sci Technol, 166-167: 373-376.

Hunter, R.A. 2007. Methane production by cattle in the tropics Letter to editor. Br J Nutr, 98: 657. Jakhmola, R.C., Pahuja, T. and Raghuvansi, S.K.S. 2010. Feeding strategies to reduce enteric methane production in ruminants: a review. Indian J Small Rumin, 16: 1-17

Jayanegara, A., Togtokhbayar, N., Makkar, HPS. and Becker, K. 2009. Tannins determined by various methods as predictors of methane production reduction potential of plants by an in vitro rumen fermentation. Anim Feed Sci Technol, 150: 230-237.

Johnson, J.M.F., Franzluebbers, A.J., Lachnicht, S. and Reicosky, D.C. 2007. Review: agricultural opportunities to mitigate greenhouse gas emissions. Environ Pollut, 150: 107-124.

Johnson, K.A. and Johnson, D.E. 1995. Methane emissions from cattle. J Anim Sci, 73: 24832492.

Kamra, N.D., Agarwal, N. and Chaudhary, L.C. 2010. Effect of tropical feeds and plants containing secondary metabolites on methane emission by ruminants. Trop Anim Health Pro, 1701: 1-23.

Kaufmann, W., Hagemeister, H. and Dirkse, G. 1980. Adaptation to changes in dietary composition, level and frequency of feeding. In: Ruckebush, Y. and Thivend, P. (Eds.). Digestive physiology and metabolism in ruminants. MTP Press. Lancaster. UK.

Klevenhusen, F., Bernasconi, S.M., Hofstetter, T., Bolotin, J., Kunz, C. and Soliva, C.R. 2009. Efficiency of monolaurin in mitigating ruminal methanogenesis and modifying $\mathrm{C}$-isotope fractionation when incubating diets of either $\mathrm{C} 3$ or $\mathrm{C} 4$ plants in a rumen simulation technique (Rusitec) system. Brit J Nutr, 18: 6, 1-11.

Knight, T.W., Molano, G., Nichols, W. and Clark, H. 2007. Effect of feeding caucasian clover, white clover, ryegrass and combinations of ryegrass and clovers on methane emissions of weather lambs. In: Greenhouse Gases and Animal Agriculture Conference. Poster Sesion 1.P1.23. Christchurch. New Zealand.

Kurihara, M., Magner, T., Hunter, R.A. and McCrabb, G.J. 1999. Methane production and energy partition of cattle in the tropics. Brit $J$ Nutr, 81 : 227-234.

Leng, RA. 2008. The potential of feeding nitrate to reduce enteric methane production in ruminants. A Report. The Department of Climate Change. Commonwealth Government of Australia. Camberra. Australia.

Lovett, D.K., McGilloway, D., Bortolozzo, A., Hawkins, M., Callan, J., Flynn, B. and O'Mara, F.P. 2005. In vitro fermentation patterns and methane production as influenced by cultivar and season of harvest of Lolium perenne L. Grass Forage Sci. 61: 9-21.

Lovett, D.K., Bortolozzo, A., Conaghan, P., O’Kiely, P. and O'Mara, F.P. 2004. In vitro total and methane gas production as influenced by rate of nitrogen application, season of harvest and perennial ryegrass cultivar. Grass Forage Sci, 59: 227-232.

Machmüller, A., Soliva, C.R. and Kreuzer, M. 2003. Effect of coconut oil and defaunation treatment on methanogenesis in sheep. Reprod Nutr Dev, 43: 41-55.

Makkar, H.P.S., Blümmel, M. and Becker, K. 1996. In vitro effects of and interactions between tannins and saponins and fate of tannins in the rumen. J Sci Food Agr, 69: 481-493.

Margan, D.E., Graham, N.M., Minson, D.J. and Searle, T.W. 1988. Energy and protein values of four forages, including a comparison of tropical and temperate species. Aust J Exp Agri, 28: 729-736.

Martin, C., Morgavi, D.P. and Doreau, M. 2009. Methane mitigation in ruminants: from microbe to farm scale. The Animal Consortium 2009. Animal, 4: 1-15.

Mbanzamihigo, L., Fievez, V., da Costa-Gomez, C., Piattoni, F., Carlier, L. and Demeyer, D. 2002. 


\section{VARGAS, CÁRDENAS, PABÓNY CARULLA}

Methane emissions from rumen of sheep fed a mixed grass-clover pasture at two fertilization rates in early and late season. Can J Anim Sci, 82: 69-77.

McAllister, T.A. and Newbold, C.J. 2008. Redirecting rumen fermentation to reduce methanogenesis. Aust J Exp Agri, 48: 7-13.

McAllister, T.A., Okine, E.K., Mathison, G.W. and Cheng, K.J. 1996. Dietary, environmental and microbiological aspects of methane production in ruminants. Can J Anim Sci, 76: 231-243.

McCaughey, W.P., Wittenberg, K. and Corrigan D. 1999. Impact of pasture on methane production by lactating beef cows. Can J Anim Sci, 79: 221-226.

McCaughey, W.P., Wittenberg, K. and Corrigan, D. 1997. Methane production by steers on pasture. Can J Anim Sci, 77: 519-524.

McCrabb, G.J. and Hunter, R.A. 1999. Prediction of methane emissions from beef cattle in tropical production systems. Aust J Agri Res, 50: 13351339.

McSweeney, C.S., Palmer, B., Bunch, R. and Krause, D.O. 2001. Effect of the tropical forage Calliandra on microbial protein synthesis and ecology in the rumen. $J$ Appl Microbiol, 90: 7888.

Mieres, J., Olivera, L., Martino, D., La Manna, A., Fernándes, E., Palermo, R. and Gremminger, H. 2002. Methane emissions from holstein heifers grazing contrasting pastures in Uruguay. http:/ /www.inia.org.uy/disciplinas/agroclima/publicaciones/ambiente/methane_uruguay.pdf (05/ 10/2012).

Misselbrook, T., Kim, E.J., Bulmer, N., Munrray, R., Scollan, N. and Chadwick, D. 2010. Evaluation of effects of grass water-soluble carbohydrate on methane emissions from grazing lambs. In: McGeough, EJ. and McGinn, S.M. (Eds.). Proceeding of the $4^{\text {th }}$ Greenhouse Gases and Animal Agriculture Conference. Greenhouse Gases and Animal Agriculture Conference. Banff, Canadá.

Moe, P.W. and Tyrrell, H.F. 1979. Methane production in dairy cows. J Dairy Sci, 62: 15831586.

Molano, G. and Clark, H. 2008. The effect of level of intake and forage quality on methane production by sheep. Aust J Exp Agri, 48: 219222.
Muetzel, S., Knight, T.W., Hoskin, S.O., Molano, G., Maclean, S., Silva-Villacorta, D. and Clark, $\mathrm{H}$. 2009. Level of intake and physiological state influences methane emissions from sheep fed fresh pasture. En: Chilliard, Y., Glasser, F., Faulconnier, Y., Bocquier, F., Vissier, I. and Doreau, M. (Eds.). Ruminant physiology, digestion metabolism and effects of nutrition on reproduction and welfare. Proceedings of the XI International Symposium on Ruminant Physiology. Clermont-Ferrand. France.

Murphy, M.R., Baldwin, R.L. and Koong, L.J. 1982. Estimation of stoichiometric parameters for rumen fermentation of roughage and concentrate diets. J Animal Sci, 55: 411-421.

Navarro-Villa, A., O’Brien, M., López, S., Boland, TM. and O'Kiely, P. 2011. In vitro rumen methane output of red clover and perennial ryegrass assayed using the gas production technique (GPT). Anim Feed Sci Technol, 168: 152-164.

Neely, C., Bunning, S. and Wilkes, A. 2009. Review of evidence on drylands pastoral systems and climate change. Implications and opportunities for mitigation and adaptation. Land and Water Discussion Paper 8. FAO. Roma, Italia.

Niggli, U., Fliebbach, A., Hepperly, P. and Scialabba, N. 2009. Low greenhouse gas agriculture: Mitigation and adaptation potential of sustainable farming systems. FAO. Rev 2-2009.

O'Mara, F.P., Beauchemin, K.A., Kreuzer, M. and McAllister, T.A. 2008. Reduction of greenhouse gas emissions of ruminants through nutritional strategies. In: Rowlinson, P., Steele, M. y Nefzaoui, A. Proceeding International Conference. Livestock and Global Climate Change 2008. Hammamet. Tunisia.

Orpin, C.G. and Joblin, K.N. 1997. The rumen anaerobic fungi. In: Hobson, P.N. and Stewart, C.S. (Eds.). The rumen microbial ecosystem. Blackie Academic \& Professional. London, UK. p. 174.

Palmquist, D.L. and Jenkins, T.C. 2003. Challenges with fats and fatty acid methods. J Anim Sci, 81: 3250-3254.

Patra, A.K. and Saxena, J. 2010. Review: A new perspective on the use of plant secondary metabolites to inhibit methanogenesis in the rumen. Phytochemistry, 71: 1198-1222.

Pinares-Patiño, C.S., Waghorn, G.C., Machmüller, A., Vlaming, B., Molano, G., Cavanagh, A. and 


\section{METANO ENTÉRICO ENSISTEMAS PASTORILES}

Clark, H. 2007a. Methane emissions and digestive physiology of non-lactating dairy cows fed pasture forage. Can J Anim Sci, 86: 601613.

Pinares-Patiño, C.S., D’Hour, P., Jouany, J.P. and Martin, C. 2007b. Effects of stocking rate on methane and carbon dioxide emissions from grazing cattle. Agr Ecosystems Environ, 121: 30-46.

Pinares-Patiño, C.S., Ulyatt, M.J., Waghorn, G.C., Lassey, K.R., Barry, T.N., Holmes, C.W. and Johnson, D.E. 2003a. Methane emissions by alpaca and sheep fed on lucerne hay or grazed on pastures of perennial ryegrass/white clover or birdsfoot trefoil. J Agr Sci, 140: 215-226.

Pinares-Patiño, C.S., Baumont, R. and Martin, C. 2003b. Methane emissions by Charolais cows grazing a monospecific pasture of timonthy at four stages of maturity. Can J Anim Sci, 83: 769-777.

Pinares-Patiño, C.S., Ulyatt, M.J., Lassey, K.R., Barry, T.N. and Holmes, C.W. 2003c. Rumen function and digestion parameters associated with differences between sheep in methane emissions when fed chaffed lucerne hay. J Agr Sci, 140: 205-214.

Pinares-Patiño, C.S., Ulyatt, M.J., Lassey, K.R., Barry, T.N. and Holmes, C.W. 2003d. Persistence of differences between sheep in methane emission under generous grazing conditions. $J$ Agr Sci, 140: 227-233.

Primavesi, O., Frighetto, R.T.S., Pedreira, M.S., Lima, M.A. and Berchielli, T.T. 2004. Metano entérico de bovinos leiteiros em condições tropicais brasileiras. Pesq Agropec Bras, 39: 277-283.

Primavesi, O., Frighetto, R.T.S., Pedreira, M.S., Lima, M.A., Berchielli, T.T. and Rodrigues, A.A. 2003. Low-fiber sugarcane to improved meat production with less methane emissions in tropical dry season. http: //www.coalinfo.net.cn/ coalbed/meeting/2203/papers/agriculture/ AF066.pdf (05/10/2012).

Purcell, P.J., O'Brien, M., Navarro-Villa, A., Boland, T.M., McEvoy, M., Grogan, D. and O'Kiely, P. 2012. In vitro rumen methane output of perennial ryegrass varieties and perennial grass species harvested throughout the growing season. Grass Forage Sci, doi: 10.1111/j.13652494.2011.00845.x
Purcell, P.J., O’Brien, M., Boland, T.M., O’Donovan, M. and O'Kiely, P. 2011. Impacts of herbage mass and sward allowance of perennial ryegrass sampled throughout the growing season on in vitro rumen methane production. Anim Feed Sci Technol, 166-167: 405-411.

Salisbury, FB. and Ross, CW. 2000. Fisiología de las plantas 2. Bioquímica vegetal. Traducción Alonso, JM. Thomson Editores Spain. Madrid. España. pp. 359-374.

Smith, A.H., Zoetendal, E.G. and Mackie, R.I. 2005. Bacterial mechanisms to overcome inhibitory effects of dietary tannins. Microb Ecol, 50: 197-205.

Solomon, S.D., Qin, M., Manning, R.B., Alley, T., Berntsen, N.L., Bindoff, Z., Chen, A., Chidtahisong, J.M., Gregory, G.C., Hegerl, M., Heimann, B., Hewitson, B.J., Hoskins, F., Joos, J., Jouzel, V., Kattsov, U., Lohmann, T., Matsuno M., Molina, N., Nicholls, J., Overpeck, J., Raga, G., Ramaswamy, J., Ren, M., Rusticucci, R., Somerville, T.F., Stocker, P., Whetton, R.A., and Wratt, D. 2007. Technical Summary. In: Solomon, S., Quin, D., Manning, M., Chen, Z., Marquis, K.B., Averyt, M. and Miller, H.L. (Eds.). Climatate Changue 2007: The Physical Science Basis. Contributioon of Working Group I to the $4^{\text {th }}$ Assessment Report of the Intergovernmental Panel on Climate Changue. Cambridge University Press. Cambridge, UK.

Sun, X.Z., Hoskin, S.O., Zhang, G.G., Molano, G., Muetzel, S., Pinares-Patiño, C.S., Clark, H. and Pacheco, D. 2011a. Sheep fed forage chicory (Cichorium intybus) or perennial ryegrass (Loliumm perenne) have similar methane emissions. Anim Feed Sci Technol, 172: 217225.

Sun, X.Z., Hoskin, S.O., Muetzel, S., Molano, G. and Clark, H. 2011b. Effects of forage chicory (Cichorium intybus) and perennial ryegrass (Loliumm perenne) on methane emissions in vitro and from sheep. Anim Feed Sci Technol, 166-167: 391-397.

Sun, X.Z., Waghorn, G.C. and Clark, H. 2010. Cultivar and age of regrowth effects on physical, chemical and in sacco degradation kinetics of vegetative perennial ryegrass (Lolium perenne L). Anim Feed Sci Technol, 155: 172-185.

Swainson, N.M. 2011. Methane emissions and mitigation technologies in cattle, sheep and red 


\section{VARGAS, CÁRDENAS, PABÓNY CARULLA}

deer. Doctor of Philosophy Thesis. Massey University. Palmerston North. New Zealand.

Tavendale, M.H., Meagher, L., Pacheco, D., Walker, N., Attwood, G.T. and Sivakumaran, S. 2005. Methane production from in vitro rumen incubations with Lotus pedunculatus and Medicago sativa, and effects of extractable condensed tannin fractions on methanogenesis. Anim Feed Sci Technol, 123-124: 403-419.

Taweel, H.Z., Tas, B.M., Smit, H.J., Elgersma, A., Dijkstra, J. and Tamminga, S. 2005. Effects of feeding perennial ryegrass with an elevated concentration of water-soluble carbohydrates on intake, rumen function and performance dairy cows. Anim Feed Sci Technol, 121: 243256.

Theodoridou, K., Aufrère, J., Niderkorn, V., Andueza, D., Morvan, A.L., Picard, F. and Baumont, R. 2011. In vitro study of the effects of condensed tannins in sainfoin on the digestive process in the rumen at two vegetation cycles. Anim Feed Sci Technol, 170: 147-159.

Tiemann, T.T., Avila, P., Ramírez, G., Lascano, C.E., Kreuzer, M. and Hess, H.D. 2008a. In vitro ruminal fermentation of tanniferous tropical plants: Plant-specific tannin effects and counteracting efficiency of PEG. Anim Feed Sci Technol, 146: 222-241.

Tiemann, T.T., Lascano, C.E., Wettstein, H.R., Mayer, A.C., Kreuzer, M. and Hess, H.D. 2008b. Effect of the tropical tannin-rich shrub legumes Calliandra calothyrsus and Flemingia macrophylla on methane emission and nitrogen and energy balance in growing labs. Animal, 2: 790-799.

Van Dorland, H.A., Wettstein, H.R., Leuenberger, H. and Kreuzer, M. 2007. Effect of supplementation of fresh and ensiled clovers to ryegrass on nitrogen loss and methane emission in dairy cows. Liv Sci, 111: 57-69.

Van Soest, P.J. 1994. Nutritional ecology of the ruminant. $2^{\mathrm{a}}$ edition. Cornell University Press. USA. pp. 253-316.

Waghorn, G.C. and Hegarty, R.S. 2011. Lowering ruminant methane emissions through improved feed conversion efficiency. Anim Feed Sci Tech, 166-167: 291-301.
Waghorn, G. 2008. Beneficial and detrimental effects of dietary condensed tannins for sustainable sheep and goat production. Progress and challenges. Anim Feed Sci. 147: 116-139.

Waghorn, G.C. and Clark, D.A. 2006. Greenhouse gas mitigation opportunities with immediate application to pastoral grazing for ruminants. Int Congr Series, 1293: 107-110.

Waghorn, G.C., Tavendale, M.H. and Woodfield, D.R. 2002. Methanogenesis from forages fed sheep. P New Zeal Grass Assoc, 64: 167-171.

Wang, C.J., Wang, S.P. and Zhou, H. 2009. Influences of flavomycin, ropadiar, and saponin on nutrient digestibility, rumen fermentation, and methane emission from sheep. Anim Feed Sci Tech, 148: 157-166.

Williams, Y.J., Walker, G.P., Doyle, P.T., Egan, A.R. and Stockdale, C.R. 2005. Rumen fermentation characteristics of dairy cows grazing different allowance of Persian clover- or perennial ryegrass-dominant swards in spring. Aust $J$ Exp Agri, 45: 665-675.

Williams, A.G. and Coleman, G.S. 1997. The rumen Protozoa. In: Hobson, P.N. and Stewart, C.S. (Eds.). The rumen microbial ecosystem. Blackie Academic \& Professional. London, UK. p. 82.

Wilson, J.R. 1993. Organization of forage plant tissues. In: Jung, H.G., Buxton, D.R., Hatfield R.D., and Ralph, J. (Eds.). Forage cell wall structure and digestibility. Winsconsin, USA. pp. 1-27.

Wims, C.M., Deighton, M.H., Lewis, E., O'Loughlin, B., Delaby, L., Boland, T.M. and O'Donovan, M. 2010. Effect of pregrazing herbage mass on methane production, dry matter intake and milk production of grazing dairy cows during the mid-laction period. A climate for change, ppportunities for carbon-efficient farming. Association of Applied Biologists. Dublin, Ireland.

Woodward, S.L., Waghorn, G.C., Ulyatt, M.J. and Lassey, K.R. 2001. Early indications that feeding Lotus will reduce methane emissions form ruminants. Proc New Zeal Soc An, 61: 23-26.

Zeleke, A.B., Clément, C., Hess, H.D., Kreuzer, M. and Soliva, C.R. 2006. Effect of foliage from multi-purpose trees and a leguminous crop residue on in vitro methanogenesis and ruminal N use. Int Congr Series, 1293: 168-171. 